\title{
Physical characteristics of indigestible solids affect emptying from the fasting human stomach
}

\author{
B MEYER, C BEGLINGER, M NEUMAYER, AND G A STALDER \\ From the Division of Gastroenterology, University Hospital, Basel, Switzerland
}

SUMMARY Gastric emptying of indigestible solids depends on their size. It is not clear whether physical characteristics other than particle size affect emptying of indigestible solids from the fasting human stomach. We studied gastric emptying of three differently shaped particles, (cubes, spheres, rods) of either hard or soft consistency during the fasting state in human volunteers. The shape of indigestible particles did not affect their emptying. The area under the gastric emptying curve (AUC: particles $\times$ hour) was for hard cubes $24 \cdot 7(2 \cdot 2)$, for hard spheres $27 \cdot 9(1 \cdot 6)$, for hard rods $26 \cdot 9(2 \cdot 7)$. All soft particles emptied faster than their identically shaped hard counterparts, but there was no difference among the three shapes (AUC for soft cubes: $29 \cdot 2(3 \cdot 0)$, for soft spheres 32.0 (1.8), for soft rods $34 \cdot 1(1 \cdot 2)$. If gastric emptying of hard and soft particles was compared independently of their shape, soft particles emptied significantly faster than hard ones: AUC $31.8(1.2) v 26.5(1.3)$ $(p<0 \cdot 01)$. In conclusion, the consistency but not the shape significantly affects gastric emptying. Specific physical characteristics other than size and shape may affect gastric emptying of indigestible particles which may be of importance in the design of drugs.

Gastric emptying of indigestible solid particles differs considerably depending on whether they are ingested during the fasting state without any food or ingested with a meal. ${ }^{1-h}$ In the fasting state, gastric motility is organised into interdigestive motor cycles that recur periodically until being disrupted by food intake.' Phase III of the interdigestive cycle consists of a burst of contractions thought to sweep out the stomach food components and solids that were not liquefied and emptied during the fed motility state. Whereas a number of studies have dealt with gastric emptying patterns of indigestible food components or solid particles ingested with meals, ${ }^{2-5}$ little work has been done regarding the emptying of indigestible solids from the fasting human stomach. ${ }^{\circ}$

Smith and Feldman have shown that indigestible markers made from polyvinyl tubing were emptied from the fasting human stomach at similar rates whether they were 2 or $10 \mathrm{~mm}$ long. ${ }^{6}$ This, however, might have arisen because the markers had the same diameter of $2 \mathrm{~mm}$. The present study was undertaken,

Address for correspondence: Dr Beat Meyer, Division of Gastroenterology, University Hospital, CH-4031 Basel, Switzerland.

Accepted for publication 14 March 1989. therefore, to further characterise the physical properties of indigestible solids that may affect their emptying from the fasting human stomach. We studied gastric emptying of indigestible particles of different forms and consistencies that were taken during the fasting state. We assumed that ingestion of markers without food would not induce a fed motility pattern. ${ }^{6}$

\section{Methods}

\section{SUBJECTS}

Thirty three healthy, male volunteers (mean age 33 years, range 21-62) participated in these studies. The protocol was approved by the Local Ethical Committee and each subject gave written informed consent.

All studies were carried out in the morning after an overnight fast of at least 10 hours. The subjects ingested 10 solid radio-opaque markers with $50 \mathrm{ml}$ tap water. The markers used were custom made synthetic particles impregnated with barium sulphate. Three different forms of markers were used: spheres $(0.2 \mathrm{~mm})$, cubes $(2 \times 2 \times 2 \mathrm{~mm})$, and rods $(2 \times 2 \times 10 \mathrm{~mm})$. Each shape was available in two 


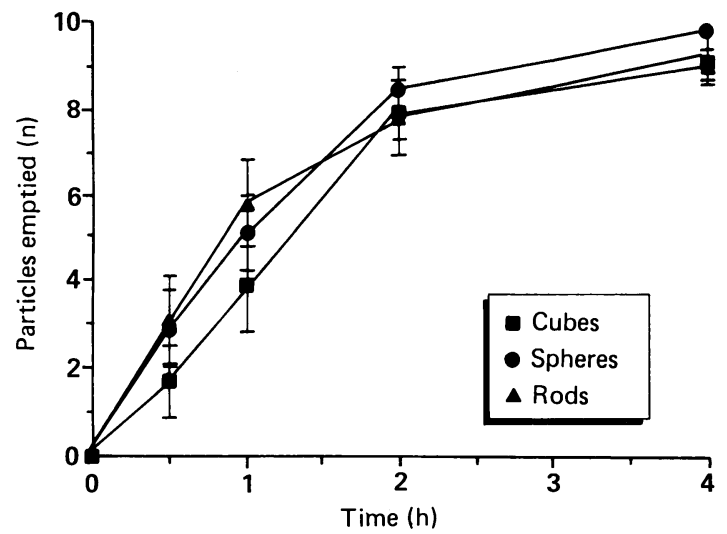

Fig. 1 Cumulative gastric emptying of three differently shaped particles of hard consistency. $(\mathbf{W}=$ cubes, $\boldsymbol{\nabla}$ spheres, $\boldsymbol{\Delta}=$ rods)

different consistencies: hard (Shore D70) markers were made from polyurethane and had a density of 1.7; soft (Shore A28) markers were made from polymerised silicone rubber and had a density of 1.4 (the consistency of the latter is comparable with that of a pencil eraser).

Twenty seven subjects were studied twice. They were divided into three groups of nine subjects who ingested 10 markers of a particular shape with either hard or soft consistency on two different days in random order. Six further subjects were studied on three different days. On each day, they ingested 10 hard markers of a particular shape in random order.

Radiographic spot films of the upper abdomen $(24 \times 30 \mathrm{~cm})$ were obtained $0 \cdot 5,1,2$, and 4 hours after marker ingestion using a high voltage technique $(100 \mathrm{KV})$ to minimise radiation exposure. The films were assessed independently by two observers (BM and $\mathrm{CB}$ ) for the number of markers retained in the stomach. Disagreement between the two observers was noted in the interpretation of five of the total 72 spot film series $(7 \%)$; these film series were reviewed and an agreement was reached. If all markers had

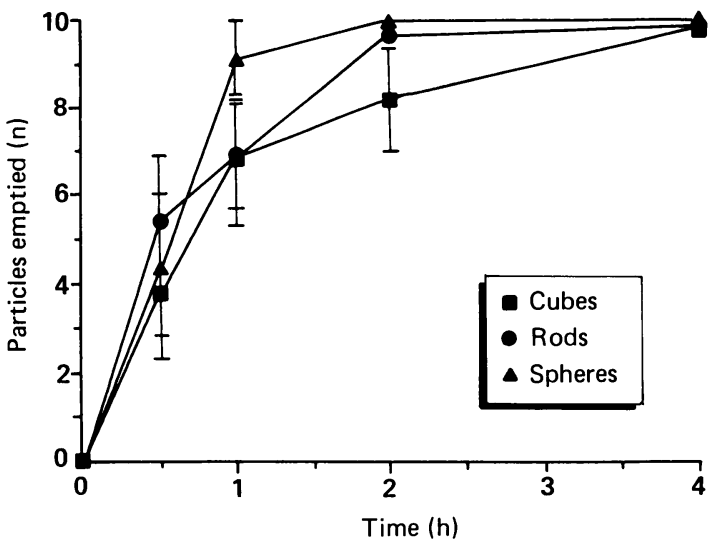

Fig. 2 Cumulative gastric emptying of three differently shaped indigestible particles of soft consistency. $(\boldsymbol{\square}=$ cubes, $\boldsymbol{\nabla}=$ spheres, $\boldsymbol{\Delta}=$ rods)

emptied from the stomach in less than four hours, subsequent radiographs were omitted. The number of markers retained in the stomach was subtracted from 10 and the results are expressed as markers emptied over time (mean (SEM)). The area under the emptying curve (particles $\times$ hour, AUC) was calculated according to the trapezoid method. Differences among treatment groups were tested for significance by ANOVA. A p value of $<0.05$ between comparisons was considered to be statistically significant.

\section{Results}

Spheres, cubes, and rods of hard consistency were emptied from the stomach at very similar rates despite marked differences in shape (Fig. 1). One hour after administration, approximately $50 \%$ of hard particles had left the stomach (Table 1) and after four hours over $90 \%$ had emptied. Figure 2 shows the emptying of the three differently shaped markers with soft consistency. Again, it is apparent that the soft particles were emptied at comparable rates

Table 1 Time dependent emptying of 10 indigestible particles (cubes, spheres, rods) of either hard or soft consistency and of all particles regardless of their shape (number of particles emptied, mean (SEM)

\begin{tabular}{|c|c|c|c|c|c|c|c|c|}
\hline \multirow{2}{*}{$\begin{array}{l}\text { Shape } \\
\text { Consistency }\end{array}$} & \multicolumn{2}{|l|}{ Cubes } & \multicolumn{2}{|l|}{ Spheres } & \multicolumn{2}{|l|}{ Rods } & \multicolumn{2}{|l|}{ All shapes } \\
\hline & Hard & Soft & Hard & Soft & Hard & Soft & Hard & Soft \\
\hline \multicolumn{9}{|l|}{ Time (min) } \\
\hline 30) & $1 \cdot 7(0 \cdot 8)$ & $3 \cdot 8(1 \cdot 5)$ & $2.9(0.9)$ & $5.4(1 \cdot 5)$ & $3 \cdot 1(1 \cdot 0)$ & $4 \cdot 4(1 \cdot 6)$ & $2.6(0.5)^{*}$ & $4.6(0.9)$ \\
\hline 60) & $3.9(1 \cdot 1)$ & $6.9(1 \cdot 2)$ & $5 \cdot 1(0 \cdot 9)$ & $6.8(1.2)$ & $5 \cdot 8(1 \cdot 1)$ & $9 \cdot 1(() \cdot 9)$ & $4.9(0 \cdot 6)^{\dagger}$ & $7 \cdot 6(0 \cdot 6)$ \\
\hline 120 & $7.9(0 \cdot 6)$ & $8 \cdot 2(1 \cdot 2)$ & $8.5(0.5)$ & $9 \cdot 6(0 \cdot 2)$ & $7 \cdot 8(0.9)$ & $10(0)$ & $8.1(0 \cdot 4)^{*}$ & $9 \cdot 3(0.4)$ \\
\hline 240 & $9 \cdot()(0 \cdot 4)$ & $9 \cdot 8(0 \cdot 2)$ & $9 \cdot 8(0 \cdot 1)$ & $9 \cdot 8(0 \cdot 2)$ & $9 \cdot 3(0 \cdot 6)$ & $10(0)$ & $9 \cdot 4(0 \cdot 2)$ & $9.9(0 \cdot 1)$ \\
\hline
\end{tabular}

${ }^{*} \mathrm{p}<0 \cdot\left(05 ;{ }^{\dagger} \mathrm{p}<0 \cdot(01\right.$. 


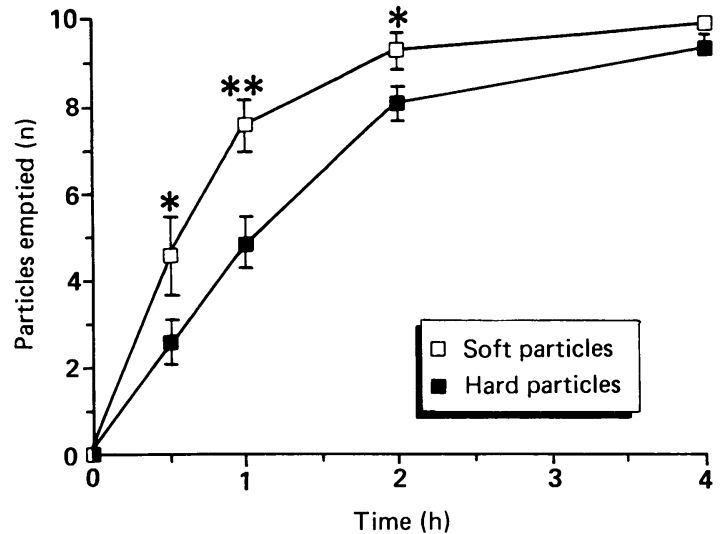

Fig. 3 Cumulative gastric emptying of indigestible particles of either hard (closed symbols) or soft (open symbols) consistency regardless of particle shape. $* * p<0.01 \quad * p<0.05$

despite different shapes. In contrast with hard particles, however, markers with soft consistency were emptied more rapidly. One hour after administration, approximately $75 \%$ of the soft markers had emptied (Table 1) and after two hours up to $100 \%$ of soft particles had left the stomach. The area under the emptying curve is given for each type of marker in Table 2. Owing to a quite large variability because of single studies where subjects emptied either type of particle very rapidly, the differences in Table 1 and Table 2 between hard and soft markers of particular shapes did not reach statistical significance. Because the shape of the markers did not influence their emptying, we assessed emptying independently of the form of the markers and compared emptying rates of soft and hard markers (Table 1, last column). Figure 3 shows the emptying curves for all hard and all soft particles. Regardless of the shape, soft particles emptied statistically significantly $(p<0 \cdot 01)$ faster than hard ones, resulting also in a significantly larger AUC (Table 2, bottom).

Table 2 Area under emptying curve ( $A U C$, particles $\times$ hour) for cubes, spheres, and rods of either hard or soft consistency and for all particles regardless of their shape

\begin{tabular}{lll}
\hline & \multicolumn{2}{l}{$A U C$} \\
\cline { 2 - 3 } Shape & Hard & Soft \\
\hline Cubes & $24 \cdot 7(2 \cdot 2)$ & $29 \cdot 2(3 \cdot 0)$ \\
Spheres & $27 \cdot 9(1 \cdot 6)$ & $32 \cdot 0(1 \cdot 8)$ \\
Rods & $26 \cdot 9(2 \cdot 7)$ & $34 \cdot 1(1 \cdot 2)$ \\
Total & $26 \cdot 5(1 \cdot 3)$ & $31 \cdot 8(1 \cdot 2)^{*}$ \\
\hline
\end{tabular}

${ }^{*} \mathrm{p}<0 \cdot 01$.

\section{Discussion}

The motor action of the stomach differs considerably depending on whether a subject is fasting or has ingested a meal.' Whereas a number of studies have investigated the relationship between physical characteristics of food components or solid particles and their emptying from the fed stomach, ${ }^{2-5 \times}$ little work has been done regarding properties of solids that may potentially influence the rate of their emptying from the stomach during interdigestive motility." Our study clearly shows that particular physical characteristics of indigestible solid particles affect the rate of their emptying from the fasting human stomach.

It has previously been observed that particles of 2 or $10 \mathrm{~mm}$ length made from polyvinyl tubing were emptied from the human stomach at similar rates." Although these markers differed considerably in their length, their diameter were identical and this might have been the reason why there was no difference in the emptying rates. In our study, we were able to show that in the fasting state, in addition to the length, the shape of solid particles also had no effect on gastric emptying. The markers we used were differently shaped, however, they also differed to some extent in their size, as shape and size are interrelated physical characteristics.

More interesting, however, is our finding that soft particles empty significantly faster from the stomach than hard ones. In dogs, it has been found that the density of indigestible markers affects the rate of their emptying. ' Polyamide spheres with a density of 1.0 emptied significantly faster than Teflon spheres of the same size, but with a density of $2 \cdot 0$. We cannot exclude that the lower density of the soft markers was responsible for the observed faster emptying, however, the difference of the densities of the two types of markers was small - that is, that of hard particles was 1.7 and that of soft particles 1.4 . Other physical characteristics may therefore have been responsible for the observed difference. Soft particles had a rubber like surface, whereas that of the hard particles was completely smooth, making it possible that forces of friction could have caused the faster emptying of softer particles. It could be argued that soft particles were compressed by the motor action of the stomach resulting in a particle size smaller than their hard counterparts which was responsible for the observed faster emptying. In spite of their consistency, however, soft particles cannot significantly be reduced in size even by forceful compression between two fingers, their consistency being approximately that of a pencil eraser. It is unclear what mechanisms are responsible for the observed difference between emptying of soft and hard particles. In the fed state, 
gastric motor action grinds and triturates solid food particles unless they are small enough to pass through the pylorus. ${ }^{-1}$ From our study, one could expect that soft food particles may pass through the pylorus more quickly than harder ones but in a recent study, gastric emptying of pancakes of either dense or light, fluffy consistency was compared and the consistency of the pancakes was found not to affect their emptying. Our study concerned emptying of indigestible particles in the fasting state and our results may therefore not be directly compared with the emptying pattern of food components. It remains unclear, however, how the stomach is able to discriminate consistency of solid particles. From clinical observations of very sharp or pinced foreign objects passing through the entire gastrointestinal tract without causing lacerations, one may assume that the mucosa exhibits some tactile properties that deserve further investigation.

Our findings that physical characteristics other than size affect gastric emptying of indigestible particles taken without food could be important in the design of drugs. Slow release formulations depend for a sustained effect mainly on gastric retention," because small bowel transit time is independent of the dosage forms. ${ }^{10}$ Pharmaceutical companies searching for ideal, slow release preparations should perhaps consider physical characteristics of their tablets other than size that may affect gastric emptying.

The authors thank Mrs Carita Frei for her expert secretarial help in preparing the manuscript. Part of this work has been published as a preliminary report in abstract form (Gastroenterology 1988; 94: A301).

\section{References}

1 Minami H, McCallum RW. The physiology and pathophysiology of gastric emptying in humans. Gastroenterology 1984; 86: 1592-610.

2 Meyer JH, Ohashi H, Jehn D. Thomson JB. Size of liver particles empticd from the human stomach. Gastroenterology 1981; 80: 1489-96.

3 Camilleri M, Malagelada JR, Brown ML, Becker G, Zinsmeister AR. Relation between antral motility and gastric emptying of solids and liquids in humans. $A m J$ Physiol 1985; 249: 580-5.

4 Holt S, Reid J, Taylor TV, Tothill P, Heading RC. Gastric emptying of solids in man. Gut 1982; 23: 292-6.

5 Feldman M. Smith HJ, Simon TR. Gastric emptying of solid radiopaque markers: Studies in healthy subjects and diabetic patients. Gastroenterology 1984; 87: 89.5902.

6 Smith HJ, Feldman M. Influence of food and marker length on gastric emptying of indigestible radiopaque markers in healthy humans. Gastroenterology 1986; 91: 1452-5.

7 Meyer JH, Dressman J, Fink A, Amidon G. Effect of size and density on canine gastric emptying of nondigestible solids. Gastroenterology 1985; 89: 805-13.

8 Houghton LA, Hickson F, Read NW. Effect of food consistency on gastric emptying in man. Gut 1987; 28: 1584-8.

9 Spiller RC. Where do all the tablets go in 1986? Gut 1986; 27: 879-85.

10 Davis SS, Hardy JG, Fara JW. Transit of pharmaceutical dosage forms through the small intestinc. Gut 1986; 27: 886-92. 